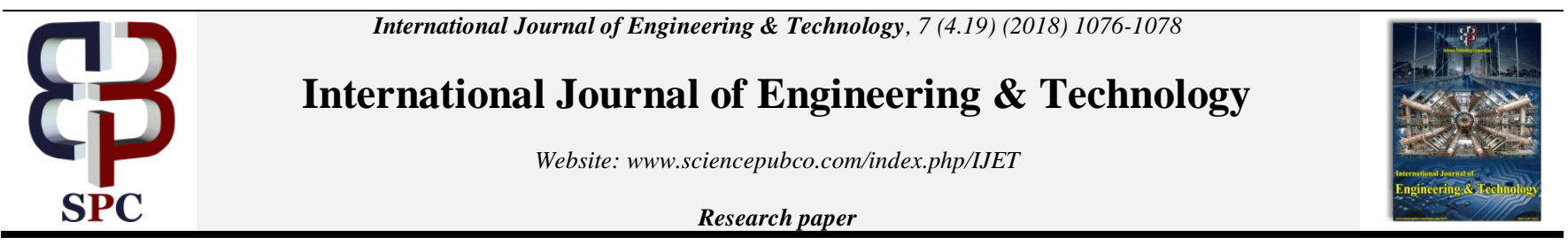

\title{
Performance of Analog Network Coded Communication with Channel Noise Assisted Gain
}

\author{
Basanti Ghanti $^{1}{ }^{*}$, Rajendra R Patil $^{2}$ \\ ${ }^{l}$ Dept. of Electronics \& Communication Engineering, Shetty Institute of Technology, Kalaburagi, India \\ ${ }^{2}$ Dept. of Electronics \& Communication Engineering, GSSSIETW, Mysore, India \\ * Corresponding author E-mail: basantighanti@gmail.com
}

\begin{abstract}
Packet forwarding by embracing interference has created a new insight in the way the interference was understood which we call it as constructive interference. In the present study the performance of Analog Network Coded Communication (ANC) based on the relay gain calculation using Channel Assisted (CA) and Channel noise Assisted (CNA) method is studied and results indicate that in the low Signal to noise ratio (SNR) and high SNR the CNA gives good performance compared to CA in terms of Sum-BER.
\end{abstract}

Keywords: Analog Network Coding, Channel Assisted, Channel Noise Assisted, Amplify-Forward, Sum-BER.

\section{Introduction}

Deploying relays for increasing the coverage area without increasing the transmitted power, creating spatial diversity through node cooperation has been an extensive research area. Prior to [1]relays were just considered for forwarding the data it was then later in the seminal work of Ahlswede the relays were used not only as forwarding nodes but also as the processing nodes. In recent times forwarding packets through network coding is an efficient way of sending packets through network. In this, packets are encoded at the relay before sending to the destination. When the signals add in the air at the relay node then it is called as physical layer network coding (PNC). This PNC is a technically feasible and appealing way to enhance the data rate of a bidirectional Relay Channel, in which the two terminals interchange the data via relay node. In a PNC system [1]relay receives the concurrently sent packets from the two source nodes. The source nodes the network coded packet generated by the relay mapping process. The end node by the advent of their own information estimate data of the other terminal using the networkcoded packet [2]. ANC can be thought as a variant of PNC where the signal is amplified by the relay instead of decoding it.

The total transmission process is split into Multiple Access (MAC) process and Broadcast (BC) process. During MAC process both the end terminals transmit their data to relay .In the $\mathrm{BC}$ process the relay sends encoded packets to the end nodes back [2] as presented in the system model. In this way the numbers of time slots required for packet transmission are reduced from four time slots to two time slots. There is an enhancement in network throughput and delay is reduced in comparison with the traditional transmission methods [3]. Network coding can also be referred as a method where signals transmitted concurrently by various nodes sum in the air [4]. Hence every node has an accumulation of these signals and noise.

As per the above discussion it is implicit that the relay processing can impact the outcome. Accordingly the relay strategy can be categorized as regenerative and non- regenerative depending on the processing method. Regenerative relay forwards the decoded data to the destination it is called as Decode - Forward (DF) relay strategy and is termed as Physical Layer Network Coding (PNC) [7]. Now if it just amplifies and forwards (AF) these signals then it is termed as Analog Network Coding (ANC) [5].Non-regenerative relaying is less complex and with smaller processing delay and actualizes the advantages of network coding .Hence we can say that the relay strategy makes a vital contribution in the overall performance of the network coded communication.

$\mathrm{AF}$ is a reasonable strategy when the relays have limited power also the AF strategy has less processing complexity and less delay [6]. The amplification gain of the relay can be based on any of the two approaches. (i) Blind or constant gain relaying; the gain of the relay is determined a priori and is fixed for all the channel conditions. (ii) Channel State Information (CSI) assisted relaying or relay with variable gain, adjusts gains depending on CSI to counterbalance the effect of fading. Full-diversity gain is obtained when the CSI is correct. The effect of obsolete CSI on relay selection of cooperative systems with $\mathrm{AF}$ and $\mathrm{DF}$ relay was studied in the earlier works. Relay with fixed gain is simple with respect to relay with variable gain. But the error rate and the ergodic capacity of the latter is superior to fixed gain relay. Hence CSI has a vital role in the outcome of cooperative communication as well as network coded systems. The determination of the relay amplification factor considering relay received noise level is called channel noise assisted (CNA) gain. If relay gain calculation does not take into account the noise level then it is termed as channel assisted (CA) gain .The Impact of CNA gain and CA gain on the BER performance of the ANC is presented in the proposed work.

\section{Literature Review}

An extensive study of the relay networks is done in [2] .A unified performance analysis presented in this paper for double- hop AF relaying with Nakagami- $m$ fading nonidentical source-to-relay and 
relay-to-destination links. Unified model for received SNR of two-hop relaying, which covers $\mathrm{CA}, \mathrm{CNA}$ and blind relay was considered.

Mazen et al. in [3] have studied the outcome of nonregenerative relays with blind relays for a double hop relay network. Also the impact of relay saturation on the outcome of system is evaluated.

Later in [4] the blind relays for the multihop scenario was studied over generalized fading channels. With generalized fading environments fresh analytical expressions are derived for the amplification factor of previously proposed semi blind relays. The moment based approach was applied to find the outage probability and the sum bit error rate for coherent and non coherent modulation schemes.

Producing a combined gain relay using CSI from both hops for a dual hop network was presented in [5]. The system outcome is analysed for Rayleigh faded links. The formulae for the moments, the average bit error probability also the outage probability of the end-to-end SNR are derived.

The semi duplex two way dual hop relay network was studied in [6]. The relay gain is based on CSI and existence of high power amplifier (HPA) non linearity is considered. The presence of interference and HPA are considered to derive end-to-end SNR. A unified analysis of the performance of for a double hop $\mathrm{AF}$ relaying is studied in [15].Considering CA, CNA and blind relays as the cases for the work an SNR model is worked out.

The impact of noise on the source nodes and the impact of interference on the relay node is studied in [16].The performance due to variation in the true channel parameters and the estimated channel parameter is validated.

\section{System Model}

The model considered in this paper is bidirectional relay network where node $A$ and node $B$ exchange data by the use of relay node which performs by applying AF protocol since the two nodes cannot communicate with each other directly.

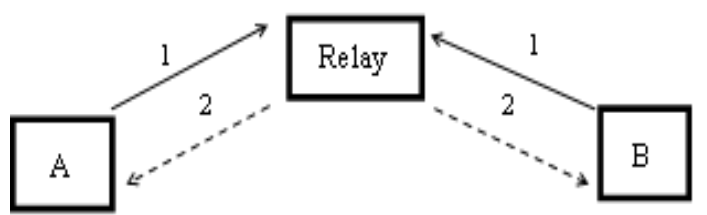

Fig. 1: Block diagram of ANC.

Let.$x_{A}$ and $x_{B}$ represent the data transmitted by node $A, B$ correspondingly and power $P_{\mathrm{A}}$ and $P_{\mathrm{B}}$. The Rayleigh channels implemented for channel from node $A$ to relay, and node $B$ to relay are and represented by $h$ and $g$ correspondingly. Noise considered is additive white Gaussian noise at node $A, B$, relay are indicated by $n_{A}-C N\left(0 . \sigma_{A}^{2}\right), n_{B}-C N\left(0 . \sigma_{B}^{2}\right), n_{r}-C N\left(0 . \sigma_{r}^{2}\right)$ respectively. Relay multiplies the signal received from node $A$ and $B$ with a gain $G$ followed by a multiplication by power $P_{r}$.

Let $\bar{\gamma}_{A}=\frac{P_{A}}{{\sigma_{r}}^{2}}, \bar{\gamma}_{B}=\frac{P_{B}}{{\sigma_{r}}^{2}}, \bar{\gamma}_{r, A}=\frac{P_{r}}{{\sigma_{A}}^{2}}, \bar{\gamma}_{r, B}=\frac{P_{r}}{\sigma_{B}{ }^{2}}$

Here, constant channels and noise are considered during these time sessions. The proposed system is illustrated in fig. 1.In the initial phase, the two source nodes concurrently send towards relay. During the consequent phase after amplifying the relay sends the signals towards two end nodes. The signal obtained at the relay in the first phase is represented as,

$r=\sqrt{P_{A}} h x_{A}+\sqrt{P_{B}} g x_{B}+n r$

After canceling the self-interference term at node $\mathrm{A}$ and $\mathrm{B}$ the signal is given by,

$y_{A}^{*}=G \sqrt{P_{r}} \sqrt{P_{B}} h g x_{B}+G \sqrt{P_{r}} h n_{r}+n_{A}$
$y_{A}^{*}=G \sqrt{P_{r}} \sqrt{P_{A}} g h x_{A}+G \sqrt{P_{r}} g n_{r}+n_{B}$

The output SNR at node A, B are represented as

$$
\begin{gathered}
\gamma_{A}=\frac{\bar{\gamma}_{r, A} \bar{\gamma}_{B}|g|^{2}|h|^{2}}{\bar{\gamma}_{r, A}|h|^{2}+\frac{1}{G^{2} \sigma_{r}^{2}}} \ldots \\
\gamma_{B}=\frac{\bar{\gamma}_{r, B} \bar{\gamma}_{A}|h|^{2}|g|^{2}}{\bar{\gamma}_{r, B}|g|^{2}+\frac{1}{G^{2} \sigma_{r}{ }^{2}}}
\end{gathered}
$$

The gain is given as $G C N A-A F$ for channel noise assisted gain, $G_{C A-A F}$ for channel assisted gain.

$$
\begin{aligned}
& G C N A-A F=\frac{1}{\sqrt{P_{A}|h|^{2}+P_{B}|g|^{2}+\zeta \sigma_{r}^{2}}} \cdots \cdots \cdots \cdots \cdots \cdots \cdots \cdots \cdots \cdots \cdots \cdots \cdots \\
& G_{C A-A F}=\frac{1}{\sqrt{P_{A}|h|^{2}+P_{B}|g|^{2}}}
\end{aligned}
$$

Where $\zeta=1$ represents gain by CNA-AF and $\zeta=0$ represents gain by CA-AF. Systems with "blind" relays do not require instantaneous CSI of the initial hop at the relay, instead apply relay of constant gain amplification resulting in a signal of changing power at relay. Systems with such blind relays are not as good as systems with CSI-assisted relays. To develop the performance measures the received SNR is represented as

$$
\gamma=\frac{\beta_{A} X Y}{\theta_{A} Y+\phi_{A} X}
$$

Where $X$ and $Y$ are random variables with exponential distribution

$\beta_{\mathrm{A}}=\bar{\gamma}_{\mathrm{r}, \mathrm{A}}, \bar{\gamma}_{\mathrm{B}}, \theta_{\mathrm{A}}=\bar{\gamma}_{\mathrm{r}, \mathrm{A}}+\bar{\gamma}_{\mathrm{A}}, \phi_{\mathrm{A}}=\bar{\gamma}_{\mathrm{B}}$ Source A

$\beta_{\mathrm{B}}=\bar{\gamma}_{r, B}, \bar{\gamma}_{A}, \theta_{\mathrm{B}}=\bar{\gamma}_{r, B}+\bar{\gamma}_{B}, \phi_{\mathrm{B}}=\bar{\gamma}_{A}$ Source B

The gain calculation by $G_{C A}-A F$ is preferred when the relay node does not have the noise statistics also it can only reciprocate the channel effects without restricting the output power of the relay. The relay amplification by applying $G C N A-A F$ method of gain calculation is used when the relay has statistics of noise and the knowledge about channel.

\section{Simulation Results}

The outcome of the ANC for CNA-AF gain and CA-AF gain are compared. The results indicate that CNA-AF achieves better sumBER than the CA-AF gain for different SNR values. 2TS indicates that two time slots are required for data transmission in ANC.

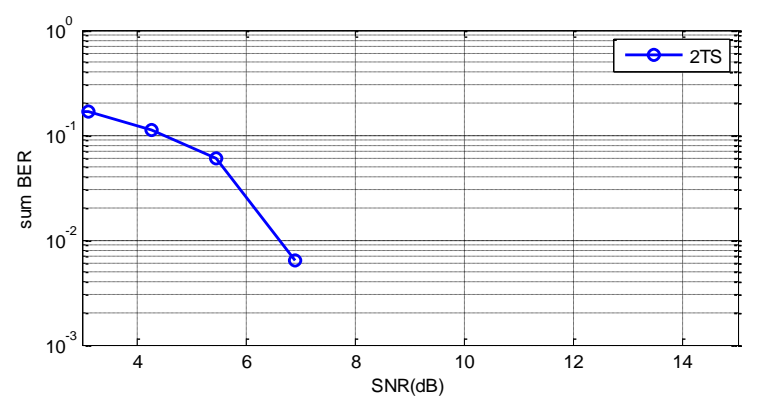

Fig. 2: Sum BER- with CNA-AF gain 


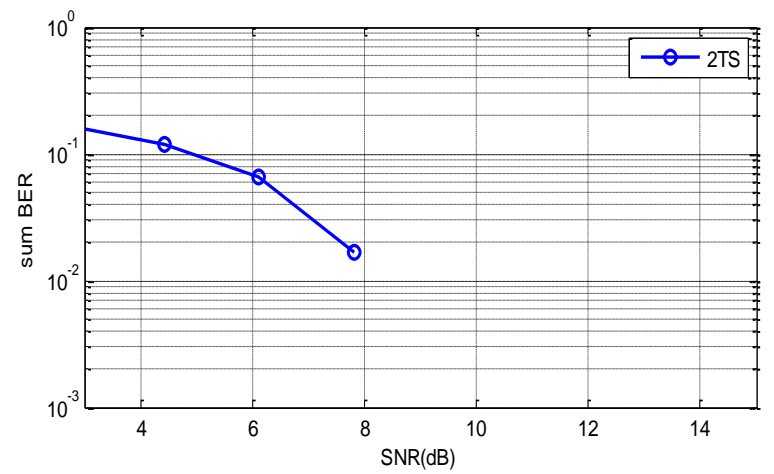

Fig. 3: Sum BER- with CA-AF gain.

\section{Conclusion}

A bidirectional relay network with $\mathrm{AF}$ relay strategy is considered. The signal received in the first time slot is amplified by the relay. The amplification factor is obtained by using either CNA-AF gain or CA-AF gain. The amplified signal is transmitted to both the nodes in the second time slot. The performance of this analog network coded communication with the above mentioned methods of finding gains is simulated and the results indicate that the performance of the CNA-AF gain is better than the CA-AF gain.

\section{References}

[1] R. Ahlswede, N. Cai, S. R. Li, and R. W. Yeung, "Network information flow," IEEE Trans. Inf. Theory, vol. 46, no. 4, pp. 1204-1216, Jul. 2000

[2] P. Anghel and M. Kaveh, "Analysis of two-hop transmission over Rayleigh fading channels," in Proc. IEEE Int. Symp. Advances in Wireless Communications, Victoria, BC, Canada, Sept. 2002, pp. $155-156$.

[3] M. O. Hasna and M. -S. Alouini, "Performance analysis of two-hop relayed transmission over Rayleigh fading channels," in Proc. IEEE Vehicular Technology Conf., Vancouver, BC, Canada, Sept. 2002, pp. 1992-1996.

[4] M. O. Hasna and M. S. Alouini, "A performance study of dual-hop transmissions with fixed gain relays," IEEE Trans. Wireles. Commun., vol. 3, no. 6, pp. 1963-1968, Nov. 2004.

[5] JG. K. Karagiannidis, "Moments-based approach to the performance analysis of equal-gain diversity in Nakagami- $m$ fading," IEEE Trans. Commun., vol. 52, no. 5, pp. 685-690, May 2004.

[6] G. K. Karagiannidis, "Performance bounds of multihop wireless communications with blind relays over generalized fading channels," IEEE Trans. Wireless Commun., vol. 5, no. 3, pp. 498503, Mar. 2006

[7] S. Katti, S. Gollakotta, and D. Katabi, "Embracing wireless interference: analog network coding," Proc. SIGCOMM, Kyoto, Japan, August, 2007

[8] Y. Song, Z. I. Sarkar, and H. Shin, "Cooperative diversity with blind relays in Nakagami-m fading channels: MRC analysis," in Vehicular Technology Conference, 2008. VTC Spring 2008. IEEE, Marina Bay, Singapore, May 2008, pp. 1196-1200.

[9] R. H. Y. Louie, Y. Li, and B. Vucetic, "Performance analysis of physical layer network coding in two-way relay channels," in Proceeding GLOBECOM'09 Proceedings of the 28th IEEE conference on Global telecommunications, 2009, pp. 2242-2247.

[10] A. Mukherjee and A. L. Swindlehurst, "Securing multi-antenna two-way relay channels with analog network coding against eavesdroppers," in 2010 IEEE 11th International Workshop on Signal Processing Advances in Wireless Communications (SPAWC), 2010, pp. 1-5.

[11] I. Mari'c, A. Goldsmith, and M. M'edard, "Analog network coding in the high-SNR regime," Proc. IEEE WiNC 2010, Boston, MA, June 2010.

[12] Z. Ding, I. Krikidis, J. Thompson, and K. K. Leung, "Physical Layer Network Coding and Precoding for the Two-Way Relay
Channel in Cellular Systems," IEEE Trans. Signal Process., vol. 59, no. 2, pp. 696-712, Feb. 2011.

[13] S. Zhang, S.-C. Liew, Q. Zhou, L. Lu, and H. Wang, "Nonmemoryless Analog Network Coding in Two-Way Relay Channel," in IEEE International Conference on Communications, 2011.

[14] T. Huang, T. Yang, J. Yuan, and I. Land, "Convergence analysis for channel-coded physical layer network coding in Gaussian twoway relay channels," in 2011 8th International Symposium on Wireless Communication Systems, 2011, pp. 849-853.

[15] M. Di Renzo, F. Graziosi, and F. Santucci, "On the performance of CSI assisted cooperative communications over generalized fading channels," in Communications, 2008. ICC '08. IEEE International Conference on, Beijing, China, May 2008, pp. 1001-1007.

[16] C. Wang, T. C.-K. Liu, and X. Dong, "Impact of channel estimation error on the performance of amplify-and-forward two-way relaying," IEEE Trans. Veh. Technol., vol. 61, no. 3, pp. 11971207, Mar. 2012.

[17] K.-A. Toshiaki, P. Popovski, and V. Tarokh, "Optimized Constellations for Two-Way Wireless Relaying with Physical Network Coding," in IEEE Journal of Selected Areas in Communications, 2015, pp. 1-15. 